\title{
Intraneural IFG-1 in Cryopreserved Nerve Isografts Increase Neural Regeneration and Functional Recovery in the Rat Sciatic Nerve
}

\author{
Sara Alicia González Porto, MD* Nieves Domenech, PhD § Francisco J. Blanco, \\ MD, PhDII Alberto Centeno Cortés, VMD\| Casto Rivadulla Fernández, PhD\#

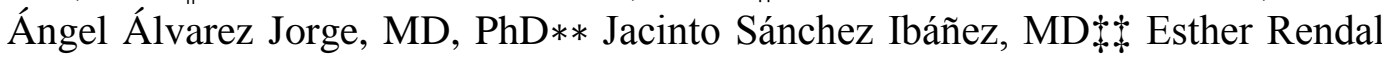 \\ Vázquez, PhDłt
}

\begin{abstract}
*Servicio de Cirugía Plástica, Hospital POVISA, Vigo, Spain; łBiobanco A Coruña, Instituto de Investigación Biomédica de A Coruña (INIBIC), Servicio Galego de Saúde (SERGAS), A Coruña, Spain; $\S C e n t r o$ de Investigación Biomédica en Red de Enfermedades Cardiovasculares (CIBERCV), Madrid, Spain; IIGrupo de Investigación de ProteómicaPBR2-ProteoRed/ISCIII-Servicio de Reumatología, Instituto de Investigación Biomédica de A Coruña (INIBIC), Complexo Hospitalario Universitario de A Coruña (CHUAC), Servicio Galego de Saúde (SERGAS), Universidade da Coruña (UDC), A Coruña, Spain; ||Centro Tecnológico de Formación XXIAC, Instituto de Investigacións Biomédicas de A Coruña (INIBIC), Servicio Galego de Saúde (SERGAS), A Coruña, Spain; \#Grupo de Neurociencia e Control Motor, NEUROcom, Facultade de Ciencias da Saúde, Departamento de Ciencias Biomédicas, Fisioterapia e Medicina, Instituto de Investigacións Biomédicas de A Coruña (INIBIC), A Coruña, Spain; **Servicio de Cirugía Plástica, Complexo Hospitalario Universitario de A Coruña (CHUAC), Servicio Galego de Saúde (SERGAS), A Coruña, Spain; HUnidad de Criobiología, Banco de Tejidos, Complexo Hospitalario Universitario de A Coruña (CHUAC), Servicio Galego de Saúde (SERGAS), A Coruña, Spain
\end{abstract}

\begin{abstract}
BACKGROUND: Insulin-like growth factor 1 (IGF-1) was found to stimulate Schwann cell mitosis. Exogenous IGF-1 may improve nerve regeneration after cryopreservation.

OBJECTIVE: To evaulate the effect of intraneural administration of IGF-1 in cryopreserved nerve isografts. METHODS: Eighteen millimeter grafts were used for bridging an $18-\mathrm{mm}$ defect in the rat sciatic nerve. A total of 57 rats were randomly divided into three groups: (1) autograft (Group 1); (2) cryopreserved isograft (Group 2); (3) cryopreserved isograft with intraneural IGF-1 administration (Group 3). 12 weeks after surgery, functional recovery (Sciatic functional index [SFI], Swing speed [SS], nerve conduction velocity [NCV], amplitude of compound motor action potentials [CMAP], and gastrocnemius muscle index [GMI]) and nerve regeneration (myelin sheath area, total fiber counts, fiber density, and fiber width) were all evaluated.

RESULTS: The intraneural injection of IGF-1 significantly improved SFI and SS at weeks 10 and 12 . There were no statistical differences between Groups 1 and 3 in any of the SFI or SS evaluations. CMAP and NCV in Group 1 were significantly higher than in Groups 2 and 3, and Group 3 had significantly higher CMAP and NCV compared to Group 2. No significant differences were found in fiber width. The number of nerve fibers, percentage of myelinated fibers, fiber density, and GMI was significantly higher in Group 1 compared to Group 2, but no significant differences were found between Groups 1 and 3.

CONCLUSION: The results show that intraneural injection of IGF-1 in an $18 \mathrm{~mm}$ cryopreserved isograft improve axonal regeneration and functional recovery.
\end{abstract}

Keywords: Albumin, Cryopreservation, Insulin-like growth factor I, Peripheral nerves, Sciatic nerve, Tissue adhesions, Tissue transplantation 
Despite the advances in microsurgery, the reconstruction of major defects in the peripheral nerve continues to be a challenge in clinical practice. ${ }^{1,2}$ The optimal treatment for a peripheral nerve defect is the use of nerve grafts from the patient him or herself (autograft). Disadvantages of this technique include limited availability and iatrogenesis in the nerve graft donor area, consisting of anesthesia or local pain. ${ }^{3,4}$ Among the alternatives to the autografts, the nerve allografts are indicated when major nerve defects are present and where autografts are insufficient. ${ }^{5}$

Cryopreservation is a method of conservation of tissues by means of cold treatment, which allows metabolic needs of tissues to be reduced to a minimum. Nerve cryopreservation is used to reduce immunogenicity within the graft, although maintaining viable Schwann cells and the scaffold for which Schwann cells can travel. ${ }^{6,7}$ This technique also permits long-term storage of nerve tissue in tissue banks, and immediate availability of cryopreserved allografts. Immunosuppression is necessary to avoid rejection of the graft, although Squintani et al ${ }^{8}$ reported the use of cryopreserved nerve allografts in the clinical setting with no adjuvant immunosuppression. ${ }^{9}$ The aim of the cryopreservation process is to keep Schwann cells viable, so that, theoretically, nerve regeneration will be greater than in acellular nerve grafts, which lack such cells. ${ }^{10}$

Apart from the scaffold for the regeneration of the nerve, we know that growth factors aid nerve regeneration. ${ }^{11}$ Insulin-like growth factor 1 (IGF-1) is a growth factor which has been shown to stimulate Schwann cell mitosis, so that it should boost the regeneration of the cryopreserved isograft. ${ }^{12}$ This growth factor improves nerve regeneration when administered locally on a daily basis or every other day. ${ }^{13-15}$ Single intraneural dosing and evaluation of the local effects of this neurotrophic factor are needed.

\section{METHODS}

\section{Animals}

The study was approved by the Institutional Review Board and all animal care complied with the Guide for the Care and Use of Laboratory Animals. Fifty-seven female adult Wistar-Lewis rats, (11-13 wk of age, weight 210-280 g) were randomized to 1 of the following groups: autograft (Group 1), cryopreserved isograft (Group 2), and cryopreserved isograft and intraneural IGF-1 (Group 3). Using the same strain of animal as donors would allow us to study the effects of cryopreservation over the nerve isograft, without interference of rejection.

In each of the three groups, 19 animals underwent sciatic nerve surgery. Nineteen rats were used as nerve isograft donors.

Inhalation anesthesia was applied, using sevoflurane. Surgery was performed under aseptic conditions using an operating microscope (Optomic OP-C12, Madrid, Spain).

\section{Preparation of Cryopreserved Nerve Isografts}

Nineteen rats underwent bilateral sciatic nerve surgery. Thirty-eight sciatic isografts were harvested. By means of a dorsal incision in both gluteal regions, biceps, and semitendinous muscles were exposed. After dissecting the sciatic nerve between these muscles, a $25-\mathrm{mm}$ nerve graft was obtained and stored individually in sterile recipients in Medium 199 with 10\% DMSO (dimethyl sulfoxide) and $4 \%$ human albumin. The cryopreservation process was performed using a cooling rate of $1^{\circ} \mathrm{C}$ per minute down to $-140^{\circ} \mathrm{C}$, the nerves being stored in nitrogen gas for 3 to 4 wk. Thawing was carried out at ambient temperature for 5 min and warm water bath for 10 min. ${ }^{16}$ 


\section{Animal Experiment}

Under inhalation anaesthesia, the rats were placed in a left lateral decubitus position. A longitudinal dorsal incision was made in the left gluteal area. An 18-mm length of sciatic nerve was removed at mid-thigh level and the nerve defect was repaired by (1) using the same autograft that has been resected in Group 1 (Auto), (2) using cryopreserved nerve isograft alone in Group 2 (Iso), or (3) using cryopreserved nerve isograft and intraneural IGF-1 injection in Group 3 (IsoIGF1; see Figure 1, which illustrates a schematic representation of the 3 groups). All grafts were $18 \mathrm{~mm}$ in length, and the repair was tension-free, using 9-0 nylon suture at each end. In Group 3, the intraneural injection of recombinant human IGF-1, $25 \mu \mathrm{L}\left(10 \mathrm{mg} / \mathrm{ml}\right.$, Increlex $\left.{ }^{\circledR} 2 \mathrm{mg}\right)$ was performed after the distal suture was made, at epineural level of the proximal graft, in order to preserve the fascicular nerve architecture, using a micropipette (Stripper ${ }^{\circledR}$ Tip, $135 \mu \mathrm{m}$, Origio, Denmark) through the proximal end of the graft.

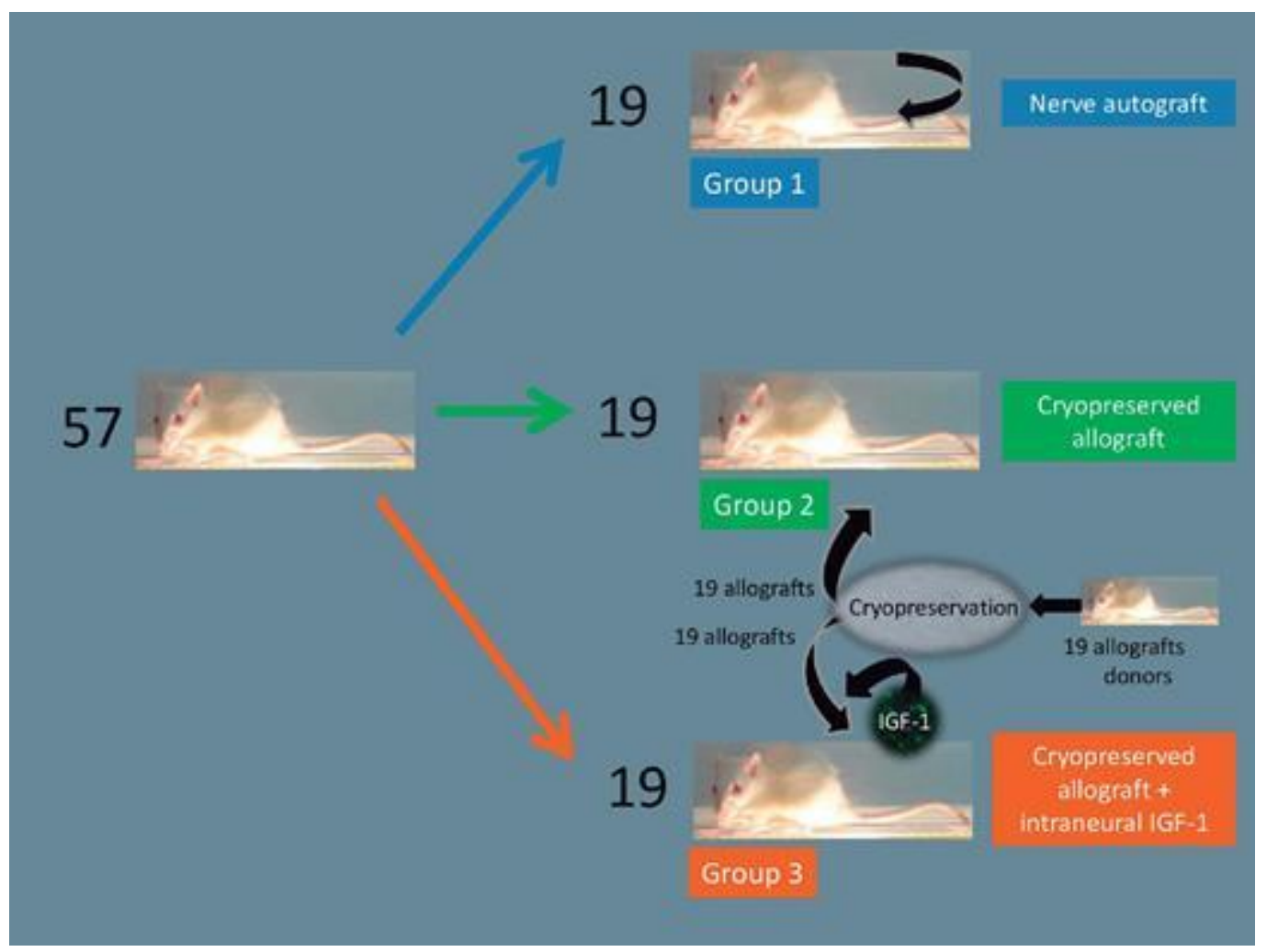

FIGURE 1. Schematic representation of the groups designed in this study. In Group 1, the gap of the sciatic nerve was bridged with an autograft from the same rat. In Group 2, cryopreserved isografts were used to reconstruct the sciatic nerve defect. In Group 3, IGF-1 was injected into the cryopreserved isografts used to reconstruct the defect. 
Animals were monitored postoperatively for infection, pain, and distress. After the functional and histological assessment at $12 \mathrm{wk}$, rats were euthanized with intrapericardial injections of Potassium Chloride (2 mEq/ml, B. Braun, Aschaffenburg, Germany).

\section{Sciatic Functional Index and Swing Speed}

Sciatic functional index (SFI) and swing speed (SS) were taken before surgery ( $0 \mathrm{wk}$ ) and after 2 , $4,6,8,10$, and $12 \mathrm{wk}$ postoperatively, according to the method described by Westerga and Gramsbergen ${ }^{17}$ and the formula proposed by Chen et al, ${ }^{18}$ respectively. The video recording technique was performed as reported by Walker et al ${ }^{19}$ and Dijkstra et al. ${ }^{20}$

The variables measured for the feet on the experimental (E) and normal side (N; expressed in milimeters) were as follows:

1. Print length (PL): Distance between heel and toe.

2. Toe spread (TS): Distance between first and fifth toes.

3. Intermediary toe spread (IT): Distance between second and fourth toes.

SFI was calculated according to the method described by De Medinacelli, ${ }^{21}$ using the modified formula described by Bain et al. ${ }^{22}$

$$
\mathrm{SFI}=-38.3\left(\frac{E P L-N P L}{N P L}\right)+109.5\left(\frac{E T S-N T S}{N T S}\right)+13.3\left(\frac{E I T-N I T}{N I T}\right)-8.8
$$

In general terms, SFI values vary from 0 (normal nerve function) to -100 (absence of sciatic function).

SS is the speed at which limbs travel between steps, and it was calculated with a manually quantitative gait analysis method, dividing the distance of the step by the time of the swing phase. The relative SS was calculated as reported by Chen et $\mathrm{al}^{18}$ as the ratio of the SS of the left hindlimb (experimental side, LSS) to the SS of the right hindlimb (normal side, RSS), and standarized against the baseline ratio (ratio of baseline SS of the left hindlimb, bLSS; to baseline SS of the right hindlimb, bRSS).

$$
\mathrm{SS}(\%)=\frac{\frac{L S S}{R S S}}{\frac{b L S S}{b R S S}} \times 100
$$

The theoretical values for relative SS vary from $0 \%$ (absence of sciatic function) to $100 \%$ (normal nerve function), being usually above $30 \%$ after sciatic nerve injury.

\section{Neural Electrophysiology}

The electrophysiological recording was determined as reported previously. ${ }^{23}$ Twelve weeks following surgery, the electrophysiology study was performed using an amplifier (AM Systems, Jaen, Spain) connected to a Power 1401 interface (Cambridge Electronic Design Limited, Cambridge, United Kingdom), to measure the compound motor action potential (CMAP) and CMAP onset latencies. The registers were stored and processed using Spike 2 software (Centre d'Estudis Demogràfics, Barcelona, Spain), by an electrophysiologist, blinded to the method of 
treatment. Under inhalation anesthesia, surgical exposure of the grafted sciatic nerve was realized. Stimulating bipolar electrodes were placed both proximal and distal to the graft, and the register electrode was placed in the gastrocnemius muscle. The distance between the bipolar electrodes was recorded. The difference of latencies between both registers was used to calculate the motor nerve conduction velocity $(\mathrm{MNCV})$.

MNCV was calculated as follows:

$$
\operatorname{MNCV}(m / s) \frac{\text { Distance between two points }(m)}{\Delta \text { latency between two points }(s)}
$$

\section{Gastrocnemius Mass Index}

After electrophysiology studies and histology nerve sampling were completed, gastrocnemius muscles from both the normal (right) and experimental (left) side were removed and weighted using an electronic balance with a precision of $1 / 10000 \mathrm{~g}$.

The gastrocnemius mass index (GMI) was calculated using the following formula:

$$
\mathrm{GMI}(\%)=\frac{\text { Wet weight of the experimental side }(\text { left })}{\text { Wet weight of the control side }(\text { right })} \times 100
$$

\section{Histological Analysis}

After the electrophysiological studies were completed, 3-mm samples were taken proximal to the distal suture line for histological analysis. Fixation with $10 \%$ formalin was performed for $24 \mathrm{~h}$, followed by gradient alcohol dehydration and paraffin inclusion. Cross-sections $4 \mu \mathrm{m}$ in width were cut with a microtome. Deparaffinization of the slices was performed at $60^{\circ} \mathrm{C}$ with xylene. They were later hydrated by immersion in $100^{\circ}$ alcohol, $96^{\circ}$ alcohol and tap water, stained by Harris hematoxylin for $5 \mathrm{~min}$, rinsed in tap water, incubated in eosin staining for $1 \mathrm{~min}$, dehydrated in $96^{\circ}$ alcohol and $100^{\circ}$ alcohol and immersed in xylene. Eight hematoxylin and eosin stained slices per nerve were observed under light microscopy in order to observe inflammatory infiltrate and the general structure of the nerve. The presence and extension of perineural scar and hypervascularized tissue surrounding the graft were assessed by 2 blinded pathologists, through a semiquantitative scale.

\section{Electronic Microscopy}

Three-mm segments were harvested from both the distal graft and distal nerve, and fixed in $2.5 \%$

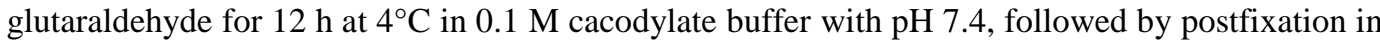
$1 \%$ osmium tetroxide, gradient acetone dehydration, and inclusion in a SPURR low-viscosity epoxy resin. The segments were cut with the ultramicrotome in $60 \mathrm{~nm}$ slices, dyed with Toluidine Blue, and observed under a JEM 1010 transmission electron microscope (Jeol, Akishima, Japan). Images were taken with a Mega View III digital camera and analyzed by analysis software (Soft Imaging System GmbH, Olympus, Shinjuku, Japan). The thickness of the myelin sheath, the area of nerve fibers, the total number of nerve fibers, the percentage of myelinated nerve fibers, and the 
density of nerve fibers were all quantified in 10 random fields of vision, magnified 2000 times, in slices from the distal nerve fragment. The pathology technician was blinded to the method of treatment.

\section{Statistical Analysis}

A descriptive analysis was performed for all the variables included, expressing them as the mean \pm SD (Standard Deviation), in addition to the $95 \%$ confidence interval. Statistical analysis was carried out with the nonparametric Kruskal-Wallis test. The Mann-Whitney $U$ test was then used as a post hoc test. The correlations between the quantitative measurements were determined by means of the Spearman correlation coefficient. SPSS 22.0 software (IBM Inc, Armonk, New York) was used for analysis. $P<.05(<5 \%)$ was considered a significant difference.

\section{RESULTS}

\section{Sciatic Functional Index and Swing Speed}

During week 2, significant differences were found between the groups in SFI $(P=.04)$, but not in SS $(P=.15$; see Figure 2 showing a schematic representation of SS). Group 2 SFI was significantly lower than that of Group $1(P=.04)$ and Group $3(P=.01)$, no differences being found between Groups 1 and $3(P=.66)$. At 2 wk there is not enough time for reinnervation in a 18 -mm graft, and the absence of significant differences in SS is more reliable than the results of SFI, which seem artefacted possibly because of the differences of the overall speed. 


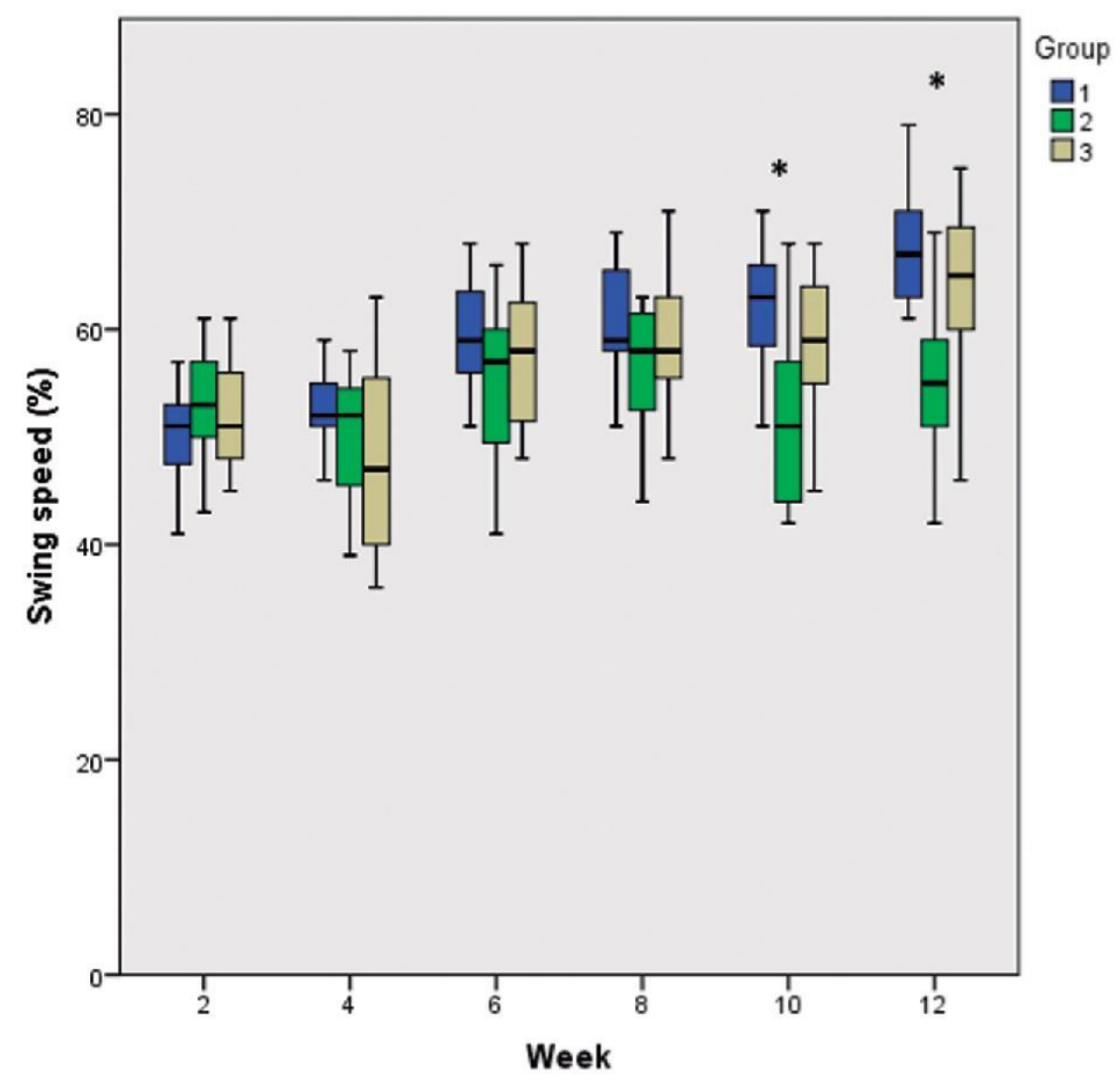

FIGURE 2. Graph showing the evolution of SS in the 3 groups. $* P<.05$ compared to the autograft. There were no significant differences between groups until week 10 . This could be explained because ten weeks is roughly the time frame expected for the regeneration front to reach the distal target. SS was superior in Group 1 compared with Groups 2 and 3 both in weeks 10 and 12. However, there are significant differences between Group 1 and Group 2 but not between Group 1 and Group 3. Group 3 significantly improved SS results in weeks 10 and 12 compared with Group 2.

In the SFI of weeks 4, 6, and 8 , no statistically significant differences were found $(P=.13, P=.06$, and $P=.08$ respectively), nor in the SS of weeks 4,6 , and $8(P=.21, P=.23$, and $P=.11$ respectively).

During weeks 10 and 12, statistically significant differences were found between the groups in the SFI $(P=.04$ and $P=.01$, respectively) and in the SS $(P<.001$ in both weeks). SFI was significantly greater in Group 1 compared with Group $2(P=.01$ in week $10, P=.001$ in week 12$)$, with no difference being found between Groups 1 and $3(P=.32$ in week $10, P=.11$ in week 12), nor between Groups 2 and $3(P=.21$ in week $10, P=.06$ in week 12). SS was also superior in Group 1 compared with Group $2(P<.001$ in both weeks 10 and 12), without significant difference between Groups 1 and $3(P=.12$ in week 10, $P=.079$ in week 12). SS in Group 3 was significantly greater compared with Group $2(P=.001$ in week $10, P<.001$ in week 12$)$. 


\section{Neural Electrophysiology}

Statistically significant differences were found between the groups in CMAP $(P=.001)$ and nerve conduction velocity (NCV) $(P=.001)$, which were significantly higher in Group 1 than in Group 2 $(P=.001$ in CMAP, $P=.001$ in NVC) and Group $3(P=.005$ in CMAP, $P=.02$ in NVC), and higher in Group 3 compared with Group $2(P=.04$ in CMAP, $P=.04$ in NVC).

\section{Gastrocnemius Mass Index}

Statistically significant differences were found between the 3 groups in GMI $(P=.001)$, significantly lower in Group 2 compared with Groups 1 and $3(P=.001$ between Groups 1 and 2, and $P=.001$ between Groups 2 and 3), no significant differences being found between Groups 1 and $3(P=.42)$.

\section{Hematoxylin and Eosin}

Preservation of epineurium and perineurium was observed in all groups, as was the presence of neovessels. Unlike the nerve's appearance at the time of the surgical intervention, (see Figure 3, which illustrates the macroscopic nerve appearance after the neurorraphy), at $12 \mathrm{wk}$, epineuralmesoneural adhesions were observed macroscopically, being observed consistently in Group 2 of the cryopreserved isografts, and present, though to a much lesser extent, in Group 1 of the autografts.
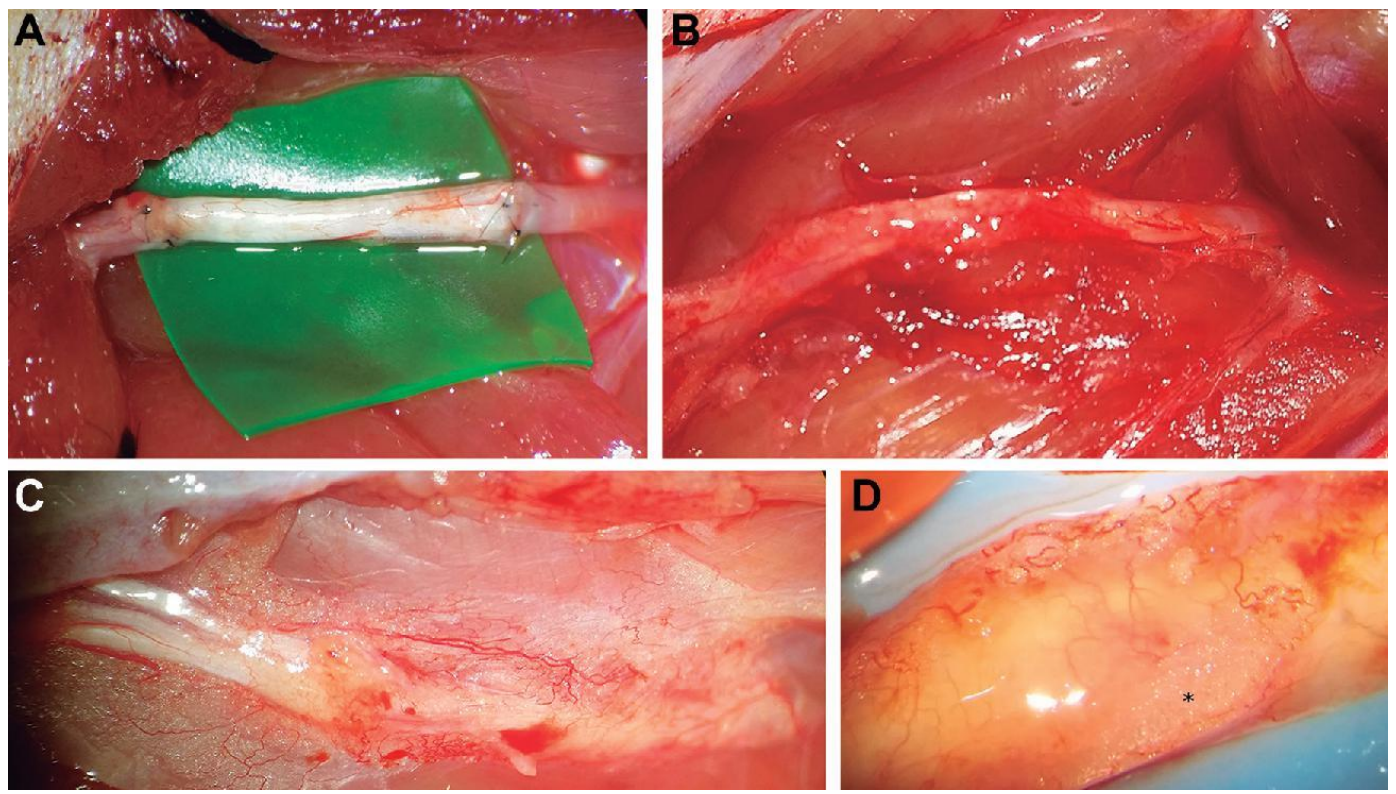

FIGURE 3. Macroscopic view of the nerve grafts. A, View of sciatic nerve at time of neurorrhaphy of graft. B, View of the cryopreserved nerve isograft 12 wk after surgery. Note the scarred aspect of the nerve, attached to the underlying muscle bed. C, View of cryopreserved nerve isograft with IGF-1 12 wk after surgery, surrounded by the shiny loose epineural tissue $(4 \times)$, and $\mathbf{D}$, shows a detail of the epineural tissue (marked with a black asterisk; 40x). 
However, in the cryopreserved isografts from Group 3, which had been injected with intraneural IGF-1, the isograft was found to be surrounded by shiny hypervascularized tissue, unattached to the surrounding muscle, permitting blunt dissection of the isograft. By using hematoxylin and eosin, it was found that this tissue was made up of subcutaneous fat, rich in numerous neovessels (see Figure 4 for further details), quite similar to the normal epineural-mesoneural tissue. The presence of perineural scarring was significantly superior in Group 2 than Group $1(P=.001)$ and $3(P=.001)$, and in Group 1 than Group $3(P=.021$; see Table 1 for further details $)$. Hypervascularized tissue was significantly more frequent in Group 3 than Group $1(P<.001)$ or Group $2(P<.001)$, and in Group 1 than Group $2(P=.013$; see Table 2 for further detail). An inverse correlation between the presence of perineural scarring and hypervascularized tissue was found, meaning that the more hypervascularized tissue the lesser scarring $(\mathrm{R}=-.708, P=.01)$.
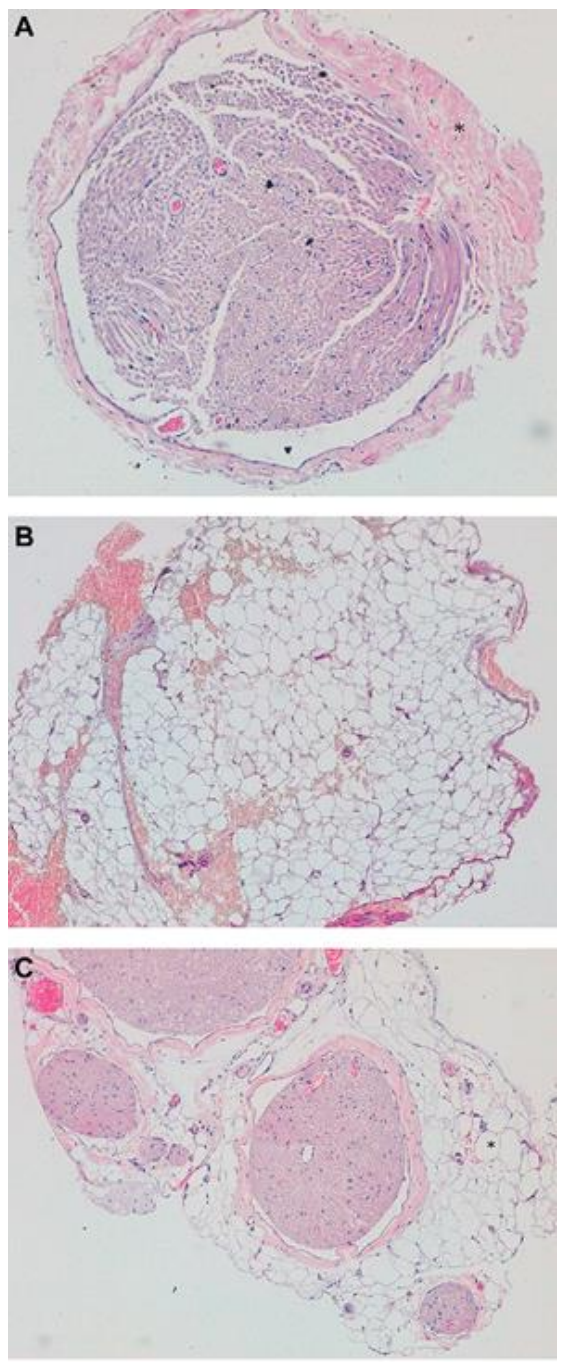

FIGURE 4. Hematoxylin and eosin. 10x. A, Microscopic view of epineural scar (marked with a black asterisk) in a cross section of the cryopreserved isograft from Group 2. B. Microscopic view of epineural tissue surrounding the cryopreserved isograft with growth factor, consisting of mature adipose cells and blood vessels. C, Microscopic view of cross-section of normal sciatic nerve. Note the similarity of normal mesoneural tissue (C, marked with a black asterisk) with the loose tissue reproduced around the cryopreserved isograft in the group with growth factor $(\mathbf{B})$. 
TABLE 1. Perineural Scarring

\begin{tabular}{|c|c|c|c|c|}
\hline & \multicolumn{4}{|c|}{ Perineural scarring } \\
\hline & $\mathbf{0}$ & + & ++ & +++ \\
\hline Autograft (Group 1) & $47.4 \%(9 / 19)$ & $47.4 \%(9 / 19)$ & $5.2 \%(1 / 19)$ & $0 \%(0 / 19)$ \\
\hline Isograft (Group 2) & $5.2 \%(1 / 19)$ & $21.1 \%(4 / 19)$ & $42.1 \%(8 / 19)$ & $31.5 \%(6 / 19)$ \\
\hline Isograft + IGF-1 (Group 3) & $84.2 \%(16 / 19)$ & $15.8 \%(3 / 19)$ & $0 \%(0 / 19)$ & $0 \%(0 / 19)$ \\
\hline Total & $45.6 \%(26 / 57)$ & $28.1(16 / 57)$ & $15.8 \%(9 / 57)$ & $10.5 \%(6 / 57)$ \\
\hline
\end{tabular}

Distribution of the rats of each group depending on the grade of perineural scarring showed in the histological analysis (0: no perineural scarring; +: mild perineural scarring; ++: moderate perineural scarring; +++: severe perineural scarring).

The percentage of rats is shown in each cell, while the number of rats in absolute figures is shown between brackets.

The table shows more intensity of the perineural scarring in Group 2, which was significantly superior than the scarring in Group $1(P=.001)$ or $3(P=.001)$. Group 1 had slightly more perineural scarring than Group $3(P=.021)$

TABLE 2. Perineural Hypervascularized Tissue

Perineural hypervascularized tissue

\begin{tabular}{lcccc}
\hline & $\mathbf{0}$ & + & ++ & +++ \\
\hline Autograft (Group 1) & $52.6 \%(10 / 19)$ & $36.8 \%(7 / 19)$ & $5.3 \%(1 / 19)$ & $5.3 \%(1 / 19)$ \\
Isograft (Group 2) & $94.7 \%(18 / 19)$ & $5.3 \%(1 / 19)$ & $0 \%(0 / 19)$ & $0 \%(0 / 19)$ \\
Isograft + IGF-1 (Group 3) & $15.8 \%(3 / 19)$ & $10.5 \%(2 / 19)$ & $26.3 \%(5 / 19)$ & $47.4 \%(9 / 19)$ \\
Total & $54.4 \%(31 / 57)$ & $17.6 \%(10 / 57)$ & $10.5 \%(6 / 57)$ & $17.5 \%(10 / 57)$ \\
\hline
\end{tabular}

Distribution of the rats of each group depending on the grade of perineural hypervascularized tissue showed in the histological analysis ( 0 : no perineural hypervascularized tissue; + : mild perineural hypervascularized tissue; ++: moderate perineural hypervascularized tissue; +++: extensive perineural hypervascularized tissue). The percentage of rats is shown in each cell, while the number of rats in absolute figures is shown between brackets.

The table reveals that the perineural hypervascularized tissue was more frequent and profuse in Group 3 . The presence of hypervascularized tissue was significantly superior in Group 3 than Group $1(P<.001)$ or Group $2(P<.001)$. Group 1 had significantly more hypervascularized tissue than Group 2 surrounding the nerve grafts

$(P=.013)$ 


\section{Electron Microscopy}

At 12 wk, Groups 1 and 3 had a larger number of myelinated nerve fibers than Group 2 (see Figure 5, which illustrates the aspect of the nerves with electron microscopy).
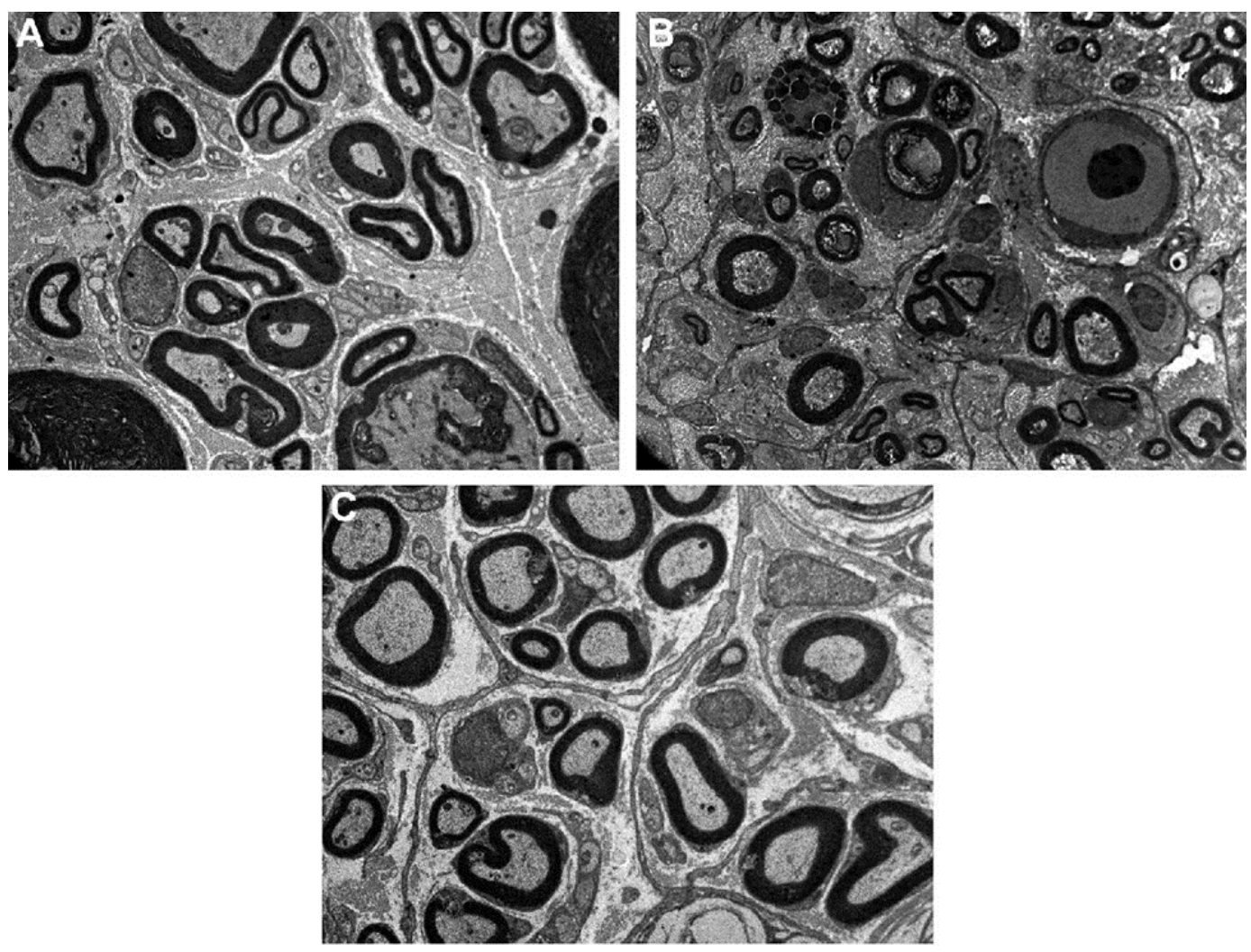

FIGURE 5. Transmission electron microscopy. Cross section of the nerve grafts (2500x). A, Corresponds to a cross section of the autograft (Group 1) in its distal portion, showing good remyelinization, limited myelin debris, a large number of axons and similar width in nerve fibers. B, Corresponds to a cross section of the cryopreserved isograft (Group 2) in its distal portion. Note the irregular remyelinization, a large amount of myelin debris and great variation in the width of the regenerated nerve fibers. $\mathbf{C}$, Corresponds to a cross section of the cryopreserved isograft with intraneural IGF-1 (Group 3) in its distal portion. The grafts with intraneural growth factor revealed more regular remyelinization, less myelin debris, and more uniform width in the myelin sheath and regenerated nerve fibers than those in the grafts from Group 2.

Differences were observed between the 3 groups regarding the thickness of the myelin sheath $(P=.001)$, which was significantly greater in Group 1 compared with Groups $2(P=.001)$ and 3 $(P=.002)$, no significant differences being observed between Groups 2 and $3(P=.15)$.

No differences were found in the average area of nerve fibers $(P=.93)$. 
The number of regenerated nerve fibers and the percentage of myelinated nerve fibers in the distal nerve differed significantly between the groups $(P=.02$ and $P=.04$, respectively). In both cases, they were significantly higher in Group 1 compared with Group $2(P=.006$ and $P=.01$, respectively), but no statistically significant differences were observed either between Groups 1 and $3(P=.27$ in total number of nerve fibers and $P=.16$ in percentage of myelinated nerve fibers), or between Groups 2 and 3 ( $P=.08$ in total number of nerve fibers and $P=.24$ in percentage of myelinated nerve fibers).

Statistically significant differences also existed between the groups in the density of nerve fibers $(P=.009)$, which was significantly higher in Group 1 compared with Group $2(P=.002)$, and in Group 3 compared with Group $2(P=.02)$, without there being any difference between Groups 1 and $3(P=.41)$.

\section{DISCUSSION}

In our experimental study, we observed that the intraneural injection of IGF-1 in the cryopreserved nerve isograft improved nerve regeneration and functional recovery, allowing similar results to those seen in the autograft group. Besides, in the group in which the growth factor was used, adipose tissue was observed around the nerve, which seems to prevent nerve scar adhesions to the surrounding muscle and reproduces the mesoneural tissue that surrounds the healthy nerve. Functional recovery parameters, such as SFI, SS, electrophysiology, and GMI, indicate significantly better results in the autograft when compared to the cryopreserved isograft. The addition of the growth factor means that outcomes improve, with the result that the cryopreserved isograft has functional recovery parameters similar to those of the autograft. In the histomorphometry, the density of nerve fibers was significantly superior when the growth factor was added. The improvement in the thickness of the myelin sheath, number of regenerated nerve fibers, and percentage of myelinated nerve fibers in the isograft with growth factor, although not statistically significant, demonstrates an enhanced nerve regeneration, similar to that of autograft. As for nerve cryopreservation, there have been few experimental studies. The nerve cryopreservation has to be taken into account when the results obtained in our study are observed, since it constitutes the reason why Group 2 has worse results than Group 1 in regeneration and functional recovery. This is due to the fact that cryopreservation means eliminating the viablity of $25 \%$ to $40 \%$ of Schwann cells, and even though the graft scaffold and most of the cell population are preserved, this process involves repercussions when nerve regeneration and functional recovery take place. ${ }^{6,7,10}$

The protocol used in this study showed $74.9 \%$ of Schwann cell viability after cryopreservation, and a significant increase of viability when albumin is added to the cryopreservation protocol of the nerve grafts. ${ }^{16}$

As for the cryoprotectants used in nerve cryopreservation, Zalewski et al ${ }^{9}$ established that the mixture of DMSO and formamide is superior to glycerol as a cryoprotectant for Schwann cells. Our group used DMSO and albumin in nerve cryopreservation, after greater viability was found when this protein was added to stabilize the membrane compared with using just DMSO. As for cooling and thawing rates, slow cooling and rapid thawing were used, as it has been shown that this method is that associated with greatest nerve fiber viability. ${ }^{24}$

The novel factor in this study was to introduce the use of IGF-1 in a single dose, administered at epineural level in the cryopreserved isograft.

IGF-1 presents a highly conserved structure among vertebrates, allowing the rat model to be suitable, even though the molecule of human IGF-1 is used. ${ }^{25}$ 
Neovessel formation is essential so that axonal regeneration can take place, and IGF-1 stimulates neoangiogenesis in nerve regeneration, ${ }^{26,27}$ in addition to inducing the differentiation of nonadipocyte precursors to fat-filled mature adipocytes. ${ }^{28}$ Along these lines and on the basis of the results of our study, we propose that the perineural adipose tissue observed in Group 3 could be related to the capacity of adipogenesis and neoangiogenesis of IGF-1. Further studies are necessary in order to find which precursors these adipocytes proceed from (satellite cells of muscle fibers, epineural or perineural cells), and which molecular mechanisms determine adipogenesis.

As for the perineural absence of adhesions which we found in Group 3, as far as we know, no publication has demonstrated this effect. As opposed to what we observed in the nerve scenario, IGF-1 has been related to the scarring of wounds, boosting neoangiogenesis, and local recruitment of macrophages. ${ }^{29,30}$ In fact, the inhibition generated by glucocorticoids on the healing of wounds could be reversed by administering IGF- $1 .{ }^{31}$

In order to find whether IGF-1 influences collagen formation locally, further studies would be necessary, with the aim of assessing the role of locally administered IGF-1 in perineural adhesions, an application which would be very interesting in relation to its use in clinical practice. We are unaware whether its local role varies according to the dose, nor the time its effect lasts.

Experimental evidence exists that IGF-1 stimulates peripheral nerve regeneration, ${ }^{11,32,33}$ encouraging both growth in nerve length and nerve branching, ${ }^{33-35}$ and also has a myogenic effect, stimulating proliferation and differentiation of myofibroblasts as well as muscle hypertrophy. ${ }^{27,36-42}$

IGF-1 has been studied in different models of lesions and nerve repair in rats. Mohammadi et $\mathrm{al}^{43}$ found improved nerve regeneration when $10 \mu \mathrm{L}$ of IGF-1 $(100 \mathrm{ng} / \mathrm{kg})$ was injected through an artery graft. Emel et $\mathrm{a}^{44}$ found increased axonal regeneration when local repeated doses of 15 $\mu \mathrm{g}$ of IGF-1 were released over crushed sciatic nerve injuries in rats. In our study, the single dose administered when surgery was performed was of $25 \mu \mathrm{L}(0.25 \mathrm{mg})$. In point of fact, one of the current limitations regarding IGF-1 is the absence of studies which examine the dose dependency of its effects. The maximum dose established for humans has been set at $0.012 \mathrm{mg} / \mathrm{kg}$ administered subcutaneously every $12 \mathrm{~h}$, being only labeled to treat growth retardance due to deficits of IGF-1. In the current piece of work, a single dose was enough to boost nerve regeneration and functional recovery. We can only postulate that continuous administration by means of percutaneous pumps could improve results. However, when it comes to clinical practice, research into the role of single intraneural administration is appealing as it is much more feasible in daily practice.

In our study, we decided to sacrifice all the rats at $12 \mathrm{wk}$, in order to avoid an increase in subgroups and the possible alpha error which could be made. The "blow-through" effect described by Brenner et $\mathrm{al}^{45}$ might determine that 4 or $8 \mathrm{wk}$ after the surgical intervention there were greater differences between the groups. Nevertheless, we consider that the existence of significant differences at $12 \mathrm{wk}$ is no justification for the sacrifice of more rats in order to study nerve regeneration 4 or 8 wk postsurgery.

The rats used in our protocol had the same haplotype, so the nerve isografts did not generate immune rejection and in our model it was unnecessary to use immunosuppression. ${ }^{46}$ This model's design was conceived exclusively to study the role of nerve cryopreservation and the addition of IGF-1 in isolation, the results being unaltered by any contribution from the immune system.

Lastly, we believe that the potential clinical applicability of IGF-1 in peripheral nerve lesions in humans needs to be grounded in fresh experimental studies which would explore its neoangiogenic and anti-scarring properties, and which could provide us with another tool with which to undertake reconstructive peripheral nerve surgery. 


\section{CONCLUSION}

This experimental study has shown that the application of a single dose of IGF-1 epineurally at the proximal end of the cryopreserved isograft improved functional recovery and sciatic nerve regeneration in the Wistar-Lewis rat model. Besides, the growth factor significantly increased epineural adipogenesis and prevented scar adhesions in the surgical bed.

\section{Disclosures}

The authors have no personal, financial, or institutional interest in any of the drugs, materials, or devices described in this article.

\section{REFERENCES}

1. Novak CB, Anastakis DJ, Beaton DE, Katz J. Patient-reported outcome after peripheral nerve injury. $J$ Hand Surg. 2009;34(2):281-287.

2. Bailey R, Kaskutas V, Fox I, Baum CM, Mackinnon SE. Effect of upper extremity nerve damage on activity participation, pain, depression, and quality of life. J Hand Surg. 2009;34(9):1682-1688.

3. Midha R. Emerging techniques for nerve repair: nerve transfers and nerve guidance tubes. Clin Neurosurg. 2006;53:185-190.

4. Battiston B, Titolo P, Ciclamini D, Panero B. Peripheral nerve defects. Hand Clin. 2017;33(3):545-550.

5. Ray WZ, Mackinnon SE. Management of nerve gaps: autografts, allografts, nerve transfers, and end-toside neurorrhaphy. Exp Neurol. 2010;223(1):77-85.

6. Ruwe PA, Trumble TE. A functional evaluation of cryopreserved peripheral nerve autografts. J Reconstr Microsurg. 1990;6(3):239-244.

7. Jensen S, Wallace MN, Dahlerup B. Cryppreservation of rat peripheral nerve segments later used for transplantation. Neuroreport. 1990;1(3):243-246.

8. Squintani G, Bonetti B, Paolin A et al. . Nerve regeneration across cryopreserved allografts from cadaveric donors: a novel approach for peripheral nerve reconstruction. J Neurosurg. 2013;119(4):907913.

9. Zalewski AA, Fahy GM, Azzam NA, Azzam RN. The fate of cryopreserved nerve isografts and allografts in normal and immunosuppressed rats. J Comp Neurol. 1993;331(1):134-147.

10. Fansa H, Lassner F, Kook PH, Keilhoff G, Schneider W. Cryopreservation of peripheral nerve grafts. Muscle Nerve. 2000;23(8):1227-1233.

11. Hansson HA. Insulin-like growth factors and nerve regeneration. Ann NY Acad Sci. 1993;692(1):161-171.

12. Fex Svenningsen A, Kanje M. Insulin and the insulin-like growth factors I and II are mitogenic to cultured rat sciatic nerve segments and stimulate $[3 \mathrm{H}]$ thymidine incorporation through their respective receptors. Glia. 1996;18(1):68-72.

13. Kanje M, Skottner A, Sjöberg J, Lundborg G. Insulin-like growth factor I (IGF-I) stimulates regeneration of the rat sciatic nerve. Brain Res. 1989;486(2):396-398.

14. Apel PJ, Ma J, Callahan M, Northam CN et al. . Effect of locally delivered IGF-1 on nerve regeneration during aging: an experimental study in rats. Muscle Nerve. 2010;41(3):335-341.

15. Corvin AP, Molinos I, Little G et al. . Insulin-like growth factor 1 (IGF1) and its active peptide (1-3)IGF1 enhance the expression of synaptic markers in neuronal circuits through different cellular mechanisms. Neurosci Lett. 2012;520(1):51-56.

16. Porto Sara Alicia González, Vázquez Esther Rendal, García Nieves Domenech, Rodríguez Alba González, Oviedo Edgar Mauricio Avellaneda, García Francisco Blanco, Gonda Carmen Arufe, Jorge Ángel Álvarez, Ibáñez Jacinto Sánchez. The addition of albumin improves Schwann cells viability in nerve cryopreservation. Cell Tissue Bank. https://doi.org/10.1007/s10561-018-9700-7.

17. Westerga J, Gramsbergen A. The development of locomotion in the rat. Dev Brain Res. 1990;57(2):163174.

18. Chen H, Du J, Zhang Y, Barnes K, Jia X. Establishing a reliable gait evaluation method for rodent studies. J Neurosci Methods. 2017;283:92-100.

19. Walker JL, Evans JM, Meade P, Resig P, Sisken BF. Gait-stance duration as a measure of injury and recovery in the rat sciatic nerve model. J Neurosci Methods. 1994;52(1):47-52. 
20. Dijkstra JR, Meek MF, Robinson PH, Gramsbergen A. Methods to evaluate functional nerve recovery in adult rats: walking track analysis, video analysis and the withdrawal reflex. J Neurosci Methods. 2000;96(2):89-96.

21. De Medinaceli L. Use of sciatic function index and walking track assessment. Microsurgery. 1990;11(2):191-192.

22. Bain JR, Mackinnon SE, Hunter DA. Functional evaluation of complete sciatic, peroneal, and posterior tibial nerve lesions in the rat. Plast Reconstr Surg. 1989;83(1):129-136.

23. Zhou L-N, Zhang J-W, Wang J-C, Lei W-L, Liu X-L, Zhou L-H. Bone marrow stromal and Schwann cells from adult rats can interact synergistically to aid in peripheral nerve repair even without intercellular contact in vitro. J Tissue Eng Regen Med. 2012;6(7):579-588.

24. Zhu Z, Qiao L, Zhao Y, Zhang S. Optimal freezing and thawing for the survival of peripheral nerves in severed rabbit limbs. Int J Clin Exp Pathol. 2014;7(11):7801-7805.

25. Rinderknecht E, Humbel RE. The amino acid sequence of human insulin-like growth factor I and its structural homology with proinsulin. J Biol Chem. 1978;253(8):2769-2776.

26. Terenghi G. Peripheral nerve injury and regeneration. Histol Histopathol. 1995;10(3):709-718.

27. Rabinovsky ED, Gelir E, Gelir S et al. . Targeted expression of IGF-1 transgene to skeletal muscle accelerates muscle and motor neuron regeneration. Faseb J. 2003;17(1):53-55.

28. Yuksel E, Weinfeld AB, Cleek $\mathrm{R}$ et al. . De novo adipose tissue generation through long-term, local delivery of insulin and insulin-like growth factor-1 by PLGA/PEG microspheres in an in vivo rat model: a novel concept and capability. Plast Reconstr Surg. 2000;105(5):1721-1729.

29. Mueller RV, Hunt TK, Tokunaga A, Spencer EM. The effect of insulinlike growth factor I on wound healing variables and macrophages in rats. Arch Surg. 1994;129(3):262-265.

30. Gimbel ML, Chelius D, Hunt TK, Spencer EM. A novel approach to reducing postoperative intraperitoneal adhesions through the inhibition of insulinlike growth factor I activity. Arch Surg. 2001;136(3):311-317.

31. Suh DY, Hunt TK, Spencer EM. Insulin-like growth factor-I reverses the impairment of wound healing induced by corticosteroids in rats. Endocrinology. 1992;131(5):2399-2403.

32. Rabinovsky ED. The multifunctional role of IGF-1 in peripheral nerve regeneration. Neurol Res. 2004;26(2):204-210.

33. Ishii DN, Glazner GW, Pu SF. Role of insulin-like growth factors in peripheral nerve regeneration. Pharmacol Ther. 1994;62(1-2):125-144.

34. Apel PJ, Ma J, Callahan M et al. . Effect of locally delivered IGF-1 on nerve regeneration during aging: an experimental study in rats. Muscle Nerve. 2010;41(3):335-341.

35. Ning H, Lin G, Fandel T, Banie L, Lue TF, Lin C-S. Insulin growth factor signaling mediates neuron-like differentiation of adipose tissue-derived stem cells. Differentiation. 2008;76(5):488-494.

36. Verdú E, Ceballos D, Vilches JJ, Navarro X. Influence of aging on peripheral nerve function and regeneration. J Peripher Nerv Syst. 2000;5(4):191-208.

37. He B, Zhu Z, Zhu Q et al. . Factors predicting sensory and motor recovery after the repair of upper limb peripheral nerve injuries. Neural Regen Res. 2014;9(6):661-672.

38. Florini JR, Ewton DZ, Coolican SA. Growth hormone and the insulin-like growth factor system in myogenesis. Endocr Rev. 1996;17(5):481-517.

39. Adams GR, McCue SA. Localized infusion of IGF-I results in skeletal muscle hypertrophy in rats. J Appl Physiol. 1998;84(5):1716-1722.

40. Musarò A, McCullagh K, Paul A et al. . Localized Igf-1 transgene expression sustains hypertrophy and regeneration in senescent skeletal muscle. Nat Genet. 2001;27(2):195-200.

41. Shavlakadze T, White JD, Davies M, Hoh JFY, Grounds MD. Insulin-like growth factor I slows the rate of denervation induced skeletal muscle atrophy. Neuromuscul Disord. 2005;15(2):139-146.

42. Nagata K, Itaka K, Baba M, Uchida S, Ishii T, Kataoka K. Muscle-targeted hydrodynamic gene introduction of insulin-like growth factor-1 using polyplex nanomicelle to treat peripheral nerve injury. $J$ Control Release. 2014;183:27-34.

43. Mohammadi R, Esmaeil-Sani Z, Amini K. Effect of local administration of insulin-like growth factor I combined with inside-out artery graft on peripheral nerve regeneration. Injury. 2013;44(10):1295-1301.

44. Emel E, Ergün SS, Kotan D et al. . Effects of insulin-like growth factor-I and platelet-rich plasma on sciatic nerve crush injury in a rat model. J Neurosurg. 2011;114(2):522-528.

45. Brenner MJ, Moradzadeh A, Myckatyn TM et al. . Role of timing in assessment of nerve regeneration. Microsurgery. 2008;28(4):265-272.

46. Shahraki M, Mohammadi R, Najafpour A. Influence of tacrolimus (FK506) on nerve regeneration using allografts: A rat sciatic nerve model. J Oral Maxillofac Surg. 2015;73(7):1438.e1-1438.e9. 


\section{Acknowledgments}

We would like to thank Alan Floyd for the review of the manuscript transcription, Catalina Sueiro and Ada Castro for their major support in the assessment of the electronic microscopy images, and Jorge Pombo Otero, Ana Teijo, and Ana Reguero for their invaluable assistance in the interpretation of the histological images. 